\title{
Prepared Material
}

National Cancer Institute

\section{Source}

National Cancer Institute. Prepared Material. NCI Thesaurus. Code C113500.

The amount of material produced by an activity. 